$\underline{\text { Review Article }}$

\title{
MESOPOROUS SILICA PARTICLES FOR DERMAL DRUG DELIVERY: A REVIEW
}

\author{
CHSAI SANJAY, VIVEK M. GHATE, SHAILA A. LEWIS*
}

Department of Pharmaceutics, Manipal College of Pharmaceutical Sciences, Manipal Academy of Higher Education, Manipal 576104, India Email: s.lewis@manipal.edu

Received: 19 Jul 2018, Revised and Accepted: 04 Sep 2018

\begin{abstract}
Mesoporous silica particles (MSP) have been reported to be applicable in diverse situations pertaining to the delivery of several drug molecules. MSP have established themselves in treating diseases with oral, dermal and parenteral modes of administration. Recently, dermal delivery using MSP have gained a considerable amount of interest owing to the increase in drug stability, permeation and ease of functionalization. MSP, in general, have a very high capability of delivering actives ranging from small molecules like drugs and amino acids to larger peptides, vaccines and antibodies. The applicability of MSP in achieving desired cosmetic and health-related outcomes depends on the careful tuning of their pore size, surface area, shape and overall physicochemical properties. This review provides comprehensive details of the recent developments in the fabrication of MSP, their characteristic features and, applications in dermal drug delivery. Studies on establishing the safety profile of MSP have also been summarized in the review.
\end{abstract}

Keywords: Mesoporous Silica, Dermal Delivery, Permeation, Pore Size, Functionalization

(C) 2018 The Authors. Published by Innovare Academic Sciences Pvt Ltd. This is an open access article under the CC BY license (http://creativecommons.org/licenses/by/4.0/) DOI: http://dx.doi.org/10.22159/ijap.2018v10i6.28633

\section{INTRODUCTION}

The word 'meso' alludesto a pore size estimate ranging from 2 to 50 nm [1]. Majorly reported mesoporous silica particles (MSP) consists of oxides of inorganic metals like silica or alumina [2]. Silicon dioxide or silica is arranged in various indentations and stacked to form different MSP characterized by their pore size, shape and pore volume. Broadly silica observed in nature are seen either in their amorphous or crystalline structures [3, 4]. MSP are a type of synthetic amorphous silica material, consisting of smallest units of less than 1 micrometer in diameter. Due to the presence of silanol groups on the surface of the MSP, they can form hydrogen bonding with various functional groups present on the entrapped molecules [5]. A recent trend seen in the utility of MSP are because of their surface chemistry mainly consisting of the hydrophilic groups that allow for sufficient solubility when given by oral and parenteral routes. A simplified surface decorated with silanol groups allows for a versatile functionalization in the development of a targeted drug delivery system [6]. Low cost of production and ease of large-scale transition make it convenient for the industries to prioritize their product profile for bulk manufacture of MSP [7]. Till date MSP have been reported to be used in drug development [5, 8], diagnosis [9] and biomarkers [10], gene delivery [11] and controlled drug release systems [12, 13]. The present review is based on the Scopus database search with keywords: mesoporous, silica, dermal, drug delivery, topical. A comprehensive data has been provided here with the search filters ranging from January 2010 to March 2018.

A wide range of MSP with a uniform pore size which included ordered, hallow and rattle types can be synthesized using different surface active agents [14]. Due to the wide availableness of various surface acting agents,various MSP structures such as Mobil Composition of Matter No. 41 and 48 (MCM-41 and MCM-48), Santa Barbara amorphous type material (SBA-15 and SBA-16), folded sheets mesoporous materials (FSM) and silica particles with bicontinuous body-centered (Ia3d) cubic symmetry(KIT-1 and KIT-6) have been synthesized [15]. Hollow or rattle type of MSP can obtain high drug loading capacity due to their low density of the outer shell of mesopores and offer greater specific area. These can be used as drug delivery systems rather than conventional systems. Toxicity and cellular interaction depend on the chemistry of the surface. Different types of functional nanoparticles such as iron oxide, gold etc. can be entrapped into the silica providing a way for targeted therapies.

MSP are chemically synthesized either in the form of ordered or unordered particles varying in their internal arrangement involving the pores and structures [16, 17]. Several ingredients though engaged in the synthesis, main components of MSP system consists of a silica precursor and structure directing surfactants. A wellcontrolled procedure and description of the characteristics can be developed for the synthesis of MSP. Extensive studies being reported worldwide on nanoparticles, a relatively newer concept of mesoporous silica nanoparticles (MSNP) have emerged. MSP and MSNP both are known to increase the solubility of drug molecules, provide greater stability to the entrapped molecules $[18,19]$ and enhance their in vivo performance.

\section{Barriers for the skin delivery}

The skin being the most prominent portion of the human body is the most robust barrier to the externally applied molecules [20]. It is composed of several layers namely the epidermis, the dermis and the hypodermis which is lined by the fatty tissues and the muscle layers. A molecule, when applied topically, has to permeate through the initial layers of dead cells called the stratum corneum. This covering is known to protect the underlying cells very efficiently from invading substances and chemicals. Being the most challenging barrier to bypass, the stratum corneum is the major hurdle to the formulating scientists. Once a molecule has crossed the initial layers of the skin, the entry into the systemic circulation and muscular tissues is solely dependent on its physicochemical properties. Apart from these, the skin is abundantly supplied by the hair follicles and sweat glands which also account for the removal of a sufficient amount of the dose applied topically [21, 22].

\section{Dermal applications of MSP}

Though several formulation strategies are available commercially like the liposomes, nanostructured lipid carriers and polymeric nanoparticle, hydrogels and topical patches there is an unmet need for developing an efficient dermal formulation till date [23]. In the purview of this, research has been directed towards the utility of using the MSP in the dermal and transdermal drug delivery applications. MSP due to their unique characteristics [24] and compatibility with the skin cells are gaining popularity in the cosmetic and dermal drug delivery applications. There are many components which affect the transdermal absorption, an active process involved in the interaction of a penetrating agent before entering into the systemic circulation. However, being in a very nascent stage successful MSP based formulation requires several factors to be considered and well-controlled parameters like their pore size, shape, and duration of exposure, distribution, the effect of other excipients and non-toxicity [25]. 


\section{Physicochemical properties}

The drug loading potential, the interaction between host and guest molecules and the action of the release profile of the drug can be achieved by proper designing and customizing the silica nanoparticles [26]. For obtaining a uniform distribution of drugs, MSP and MSNP have a free gateway for the drugs to enter despite of well-structured channels of silica particles. Biomolecules, on the other hand, are having substantial molecular weight and greater volumes such as protein molecules and genes need silica particles of larger pore size because the diameter of the pore is considered as selective size specification for the adsorption of drugs. The interaction between the guest and host is primarily a chemical bonding between silanol groups and functional groups present on the guest molecules (fig. 1). Various surface potentials, and different functional groups and lipophilic/lipophobic property of the matrix surface can be obtained by modifying the surface of the silica particles with the appropriate chemical functionalization. Permeation of the MSP through the skin is reported to be highly dependent on its physicochemical properties, not limiting to the size, agglomeration, shape, porosity, chemical groups present on the surface and the integral portions of the pores [27]. It was observed that the loading of ketoprofen took place at a higher rate in SBA-15 than in MCM-41 due to the presence of larger pore size in SBA-15 [28]. Advances in the analytical techniques have related the effects of these properties of MSP with the penetration of the active compounds. Much of the attention is focused on the aggregation of these MSP and MSPN in the physiological medium and solvents used in the routine analysis $[29,30]$. Once aggregated they are harder to redisperse and hence are unable to be clearly investigated for the purpose.

A handful of studies carried out in this regard show that despite the aggregation of MSP the skin cells can uptake the particles to a maximum particle size of $75 \mathrm{~nm}$ [31]. A slight increase in the particle size above this, do not provide for a more significant effect on the penetration of the carriers. Another notable approach to increase the permeation into the skin is the addition of permeation enhancers and functionalization of the carrier molecules. As mentioned earlier, the targeting the hair follicles is also an important strategy to increase the transdermal delivery of the drugs [32]. A study of the particles with nanometer to micrometer sizes was conducted [21] and then concluded that optimal penetration was seen in the particles in the range of $400-700 \mathrm{~nm}$ through the hair follicles. A similar study conducted showed that the penetration of the particles begins at 300 $\mathrm{nm}$ and increases until $646 \mathrm{~nm}$ after that the hair follicles were unable to uptake these particles [33]. Once the particle is in the immunogenically accessible layers of the skin, the silica particles in the said size range are also reported to cause certain types of toxicity and immunogenic reactions [34]. Chemically, the negative charges on the surface hinder the penetration into the skin cells hence functionalization would be appropriate in ascertaining the entry of MSP into the cells. A similar interaction of the MSN surface functional groups and the gram-negative and gram-positive bacteria was seen to largely inhibit the strains after being loaded with tetracycline [35].

Biocompatibility of the particles is greatly affected by the surface properties of the MSNP. Compared with the neutral and anionic analogs, cationic charged analogs are more prone to cytotoxicity and produce more immune responses [36]. Upon prolonged exposure of silanol groups to the environment, these groups may dismantle the structures of lipids, proteins, and other biological molecules. PEGylation forms a hydrophilic coating around MSNP which can significantly enhance the surface activity, biocompatibility, and circulation time (in vivo). A siRNA delivery platform was developed by coating the MSNP core with polyethyleneimine and PEG to target the heat shock protein (HSP 47) in the treatment of fibrosis. The results of the study demonstrated that the MSNP were able to reduce the levels of elevated HSP 47 to normal and also provide antioxidant properties in in vitro models of the disease [37]. Other than PEGylation, the cellular uptake, bio-distribution, excretion, and cytotoxicity can be controlled by modifications of MSNP with different functional groups such as amino, carboxyl, phenyl phosphate, methyl phosphate which includes all kinds groups that cause a change in the Zetapotentials $[29,38]$. The effect of particle shape on the toxicity of nanoparticles, drug performance, and bio distribution can be investigated by altering the diameter of the particles, different shapes and a relation between the biocompatibility and the particle shape can be obtained.

\section{MESOPOROUS SILICA NANOPARTICLES}

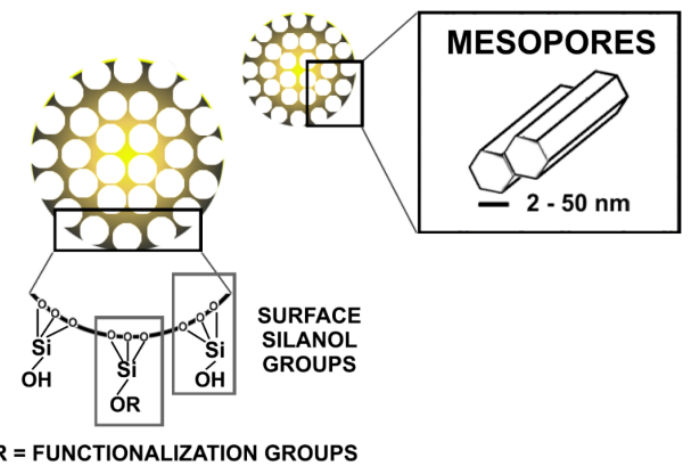

Fig. 1: Mesoporous silica nanoparticles and the surface silanol groups

\section{Excipient effect}

The topmost layer of the skin sharing the air interface is the stratum corneum. Like most other barriers, it also has an affinity for lipophilic moieties and favorably transports the substances tagged with a lipid-like compound. The explanation further moves on into the barrier preventing the entry of lipophobic or hydrophilic molecules through it. It is well-advised that a compound to be able to breach the stratum corneum has to possess considerable lipophilicity as well as slight hydrophilicity to be able to be formulated into an effective system. MSP offer a considerable amount of both these properties to the entrapped molecules thereby facilitating the entry of the entrapped molecules into the skin cells [39]. Agglomeration, on the other hand, prevents the smooth passage of the carrier systems into the cells due to the increase in the size of the particles and reduction in the surface area, which in turn elicits an immunogenic reaction on sufficient exposure. Skin in itself is a barrier that has a slightly acidic $\mathrm{pH}$ of about 6.8 thus allowing it to be highly efficient in its purpose of the activity. Therefore the ionization of the MSP and the drug moieties play a crucial role in such cases where the absorption is said to be $\mathrm{pH}$ dependent [40]. Contrary to the fact that the particles of size 300 to $600 \mathrm{~nm}$ was found to be well concentrated in the cellular systems, the particles of size above 3 micrometers were also seen to penetrate well into the dermis [41]whereas the concentration into the epidermis was possible only with an ethanolic medium (65\%). Adding onto the theories that oil and lipid-like molecules were able to bypass the stratum corneum, the coating of MSP and MSNP have been looked as an excellent strategy for the dermal or transdermal therapy. Several lipids such as lecithin and other phospholipids, and emulsion coating [42] have been coated onto the MSP, and the results have shown the extensive stability of the particles and sufficient penetration into the deeper skin layers. Substances like the retinol [43], quercetin and rutin $[44,45]$ have been explicitly studied in conjunction with the MSP coated with the oil-based emulsions. All-trans-retinol, when formulated as oil-in-water emulsions coated onto MSNP, showed that the stability was improved two times compared to the oleylamine-and lecithinstabilized droplets [43]. Flavonoids such as quercetin and rutin were loaded on to MSP functionalized with titanium. The results showed that the free radical scavenging of the ethanolic citric acid recovered and the bound flavonoids were greater than $80 \%$ [44]. Penetration in certain cases is not desirable due to the objective of treating the superficial or conditions encircling the dermis or the epidermis. In these type of applications, penetration retarders are known to serve the purpose. Recently emulsions and MSP together constituting the barrier-like function have been explored for the delivery of caffeine. 
It was shown that there was a delayed penetration of the active through the pigskin in comparison with the reference gel.

\section{other factors}

Despite taking enormous precautions and carefully selecting the ranges of the components to obtain the desired functional characteristics of MSP and MSNP for the topical administration, specific factors still exist which are out of the control of the formulators. Majorly the exfoliation, sweating and transfer to external surfaces from the skin represent the concerns for the failure of the well-formulated product [45]. Certain molecules that are to be entrapped into the MSP are sensitive to light, oxygen, and moisture. Even though the MSP are highly regarded as a system to provide stability, these conditions may pose unavoidable problems in a formulation $[46,47]$.

\section{Preparation methods of MSPs and MSNPs}

There are three ways by which functionalities are integrated with silica particles:

a) Grafting method (in which silica nanoparticles are succeeding attached by organic compounds)

b) Upon co-condensation of silica particles and the organic compounds containing silyl group (R3Si)

c) Formation of periodic mesoporous organosilicas (PMO) with the help of organic precursors which are bissilyated.

\section{Grafting method}

The major principle involved in the grafting is the reaction of organosilanes with the unreacted silanol groups present on the silica surface. Some of the silanes include organo, chloro or silazanes. The word grafting mainly alludes to sequential modification of internal pore surfaces of mesoporous silica phases with organic compounds $[48,49]$. The uniformity of the distribution of organic compounds over the silica surface depends on the size of the organic compound and the degree of tenancy, in turn, leads to the abnormal diffusion of more amount of molecules into the pores present on the silica surface which leads to the blockade of pores, thus creating an ordered or unordered pore system.

\section{Co-condesation of silica particles}

Functionalized MSP can be achieved by co-condensation of type ( $\mathrm{R}$ ' $\mathrm{O}$-3alkoxysilanes and (RO) ${ }_{4}$-alkoxysilanes where MCM-41 and SBA-15 acts as a structure-directing agent present on the pore walls so that organic residues are obtained. The primary advantage of this method is that less blockade of pores are seen due to the presence of organic functionalities which discharge into the pores of the silica particles and organic particles are uniformly distributed over the pores of silica particles when compared with the grafting process $[50,51]$. The drawbacks of this method include improper functionalization can occur, and the proportion of concentration of organic groups present on the pore walls varies leading to lower concentrations at the extreme groups compared with the initial groups. Another drawback is that upon an increase in incorporation of organic functionalities, a decrease in the size of the pore, volume of the pore and the surface area is seen.

\section{Formation of PMOs}

PMO contain a tapered pore radius because the integrated compounds of silica are formed by the organic bridges between surface active agents acting on bisilyatedorganosilica precursors and mesoporos silica particles $[52,53]$. PMO are extensively used in the preparation of active payloads.

\section{Safety concerns}

All kinds of materials applied over the skin with theintention of concentrating in the upper and deeper layers of the skin, the entry into the systemic circulation and their effect on distal organs have to be clearly addressed. Most commonly reported issues of toxicity with such formulations include the primary inflammatory responses upon contact of the actives and carriers with the epidermis which may then progress to the dermis producing allergic reactions [54]. Effects of the systemic exposure can also be seen in the distal organs upon prolonged exposure to the formulation and can also lead to certain types of cancer [55]. MSP are also not free from these kinds of complications that may be fatal in the long run [56]. Therefore it is very important to address these concerns especially in the case of MSNP clearly. The safety concerns can be adequately addressed with modern analytical techniques like the transmission electron microscopy (TEM), Raman scattering and using fluorescent probes in a confocal laser microscopy system. Apart from in vivo safety concerns, MSP are released into the environment by a variety of common industrial processes [57].

\section{CONCLUSION}

MSP and MSNP are more adaptable, flexible, and stronger than traditional drug delivery frameworks, for example, polymer nanoparticles and liposomes. The confinements of regular natural systems are low medication stacking limit, low produce, and highprice of generation. With the extraordinary mesoporous structure and high surface region, MSP as carriers have a high ability to oblige guest particles and can discharge the stacked particles in physiologic conditions. Their pore structure with capable pore size and geometry encourages a similar joining of guest particles with various sizes and surface groups. The fabrication procedure is moderately basic, and the price is fundamentally low, which is critical to satisfy the intended clinical request concerning the dermal delivery of actives.

\section{AUTHORS CONTRIBUTIONS}

All the authors have contributed equally

\section{CONFLICT OF INTERESTS}

Declared none

\section{REFERENCES}

1. Soboleva T, Zhao X, Malek K, Xie Z, Navessin T, Holdcroft S. On the micro-, meso-, and macroporous structures of polymer electrolyte membrane fuel cell catalyst layers. ACS Appl Mater Interfaces 2010;2:375-84.

2. Keshavarz M, Ahmad N. Characterization and modification of mesoporous silica nanoparticles prepared by sol-gel. J Nanopart 2013;2013:1-4. http://dx.doi.org/10.1155/2013/ 102823.

3. Lin YC, Lin LY, Gao MY, Fang YP. Mesoporous silica nanoparticles synthesized from liquid crystal display manufacturing extracts as a potential candidate for a drug delivery carrier: evaluation of their safety and biocompatibility. Int J Nanomed 2013;8:3833-42.

4. Suzuki T, Kuroda K. Deposition of single-crystalline mesoporous silica particles and the in-plane arrangement of mesocages over particles on a cleaved mica surface. J Mater Chem 2007;17:4762-7.

5. Natarajan SK, Selvaraj S. Mesoporous silica nanoparticles: importance of surface modifications and its role in drug delivery. RSC Adv 2014;4:14328-34.

6. Liberman A, Mendez N, Trogler WC, Kummel AC. Synthesis and surface functionalization of silica nanoparticles for nanomedicine. Surf Sci Rep 2014;69:132-58.

7. Wu S, Mou C, Lin H. Synthesis of mesoporous silica nanoparticles. Chem Soc Rev 2013;42:3862-75.

8. Watermann A, Brieger J. Silica nanoparticles as drug delivery vehicles in cancer. Nanomaterials (Basel, Switzerland) 2017; 7:1-17.

9. Arap W, Pasqualini R, Montalti M, Petrizza L, Prodi L, Rampazzo $\mathrm{E}$, et al. Luminescent silica nanoparticles for cancer diagnosis. Curr Med Chem 2013;20:2195-211.

10. Bouamrani A, Hu Y, Tasciotti E, Li L, Chiappini C, Liu X, et al. Mesoporous silica chips for selective enrichment and stabilization of low molecular weight proteome. Proteomics 2010;10:496-505.

11. Yu M, Niu Y, Zhang J, Zhang H, Yang Y, Taran E, et al. Sizedependent gene delivery of amine-modified silica nanoparticles. Nano Res 2016;9:291-305.

12. Gimenez C, Torre L, Gorbe M, Aznar E, Sancenon F, Murguia JR, et al. Gated mesoporous silica nanoparticles for the controlled delivery of drugs in cancer cells. Langmuir 2015;31:3753-62. 
13. Song Y, Li Y, Xu Q, Liu Z. Mesoporous silica nanoparticles for stimuli-responsive controlled drug delivery: advances, challenges, and outlook. Int I Nanomed 2017;12:87-110.

14. Teng Z, Su X, Zheng Y, Sun J, Chen G, Tian C, et al. Mesoporous silica hollow spheres with ordered radial mesochannels by a spontaneous self-transformation approach. Chem Mater 2013;25:98-105.

15. Bharti C, Nagaich U, Pal AK, Gulati N. Mesoporous silica nanoparticles in target drug delivery system: a review. Int J Pharm Invest 2015;5:124-33.

16. Chiu HY, Leonhardt H, Bein T. Synthesis and functionalization of ordered large-pore mesoporous silica nanoparticles for biomedical applications. Chem Ing Tech 2017;89:876-86.

17. Limnell T, Santos HA, Makila E, Heikkila T, Salonen J, Murzin DY, et al. Drug delivery formulations of ordered and nonordered mesoporous silica: comparison of three drug loading methods. J Pharm Sci 2011;100:3294-306.

18. Lin YS, Abadeer N, Haynes CL. Stability of small mesoporous silicananoparticles in biological media. Chem Commun 2011; 47:532-4.

19. Choi Y, Lee JE, Lee JH, Jeong JH, Kim J. A biodegradation study of SBA-15 microparticles in simulated body fluid and in vivo. Langmuir 2015;31:6457-62.

20. Richardson M. Understanding the structure and function of the skin. Nurs Times 2003;99:46-8.

21. Otberg N, Patzelt A, Rasulev U, Hagemeister T, Linscheid M, Sinkgraven R, et al. The role of hair follicles in the percutaneous absorption of caffeine. Br J Clin Pharmacol 2008;65:488-92.

22. Konradsdottir F, Ogmundsdottir H, Sigurdsson V, Loftsson T. Drug targeting to the hair follicles: a cyclodextrin-based drug delivery. AAPS PharmSciTech 2009;10:266-9.

23. Watkinson AC, Kearney MC, Quinn HL, Courtenay AJ, Donnelly RF. Future of the transdermal drug delivery market-have we barely touched the surface? Expert Opinion Drug Delivery 2016;13:523-32.

24. Ernawati L, Balgis R, Ogi T, Okuyama K. Tunable synthesis of mesoporous silica particles with unique radially oriented pore structures from tetramethyl orthosilicate via oil-water emulsion process. Langmuir 2017;33:783-90.

25. Yamada H, Urata C, Aoyama Y, Osada S, Yamauchi Y, Kuroda K. Preparation of colloidal mesoporous silica nanoparticles with different diameters and their unique degradation behavior in static aqueous systems. Chem Mater 2012;24:1462-71.

26. Lodha A, Lodha M, Patel A, Chaudhuri J, Dalal J, Edwards M, et al. Synthesis of mesoporous silica nanoparticles and drug loading of poorly water soluble drug cyclosporin A. J Pharm BioAllied Sci 2012;4(Suppl 1):S92-4.

27. Kaasalainen M, Aseyev V, Haartman E, Karaman DS, Makila E, Tenhu H, et al. Size, stability, and porosity of mesoporous nanoparticles characterized with light scattering. Nanoscale Res Lett 2017;12:74.

28. Abd-elbary, Nabarawi MAE, Hassen DH, Taha AA. Inclusion and characterization of ketoprofen into different mesoporous silica nanoparticles using three loading methods. Int J Pharm Pharm Sci 2014;6:189-91.

29. Bagwe RP, Lisa R, Hilliard LR, Tan W. Surface modification of silica nanoparticles to reduce aggregation and nonspecific binding. Langmuir 2006;22:4357-62.

30. Warther D, Jimenez CM, Raehm L, Gerardin C, Durand J, Morere $\mathrm{A}$, et al. Small sized mesoporous silica nanoparticles functionalized with mannose for retinoblastoma cell imaging. RSC Adv 2014;4:37171-9.

31. Rancan F, Gao Q, Graf C, Troppens S, Hadam S, Hackbart S, et al. Skin penetration and cellular uptake of amorphous silica nanoparticles with variable size, surface functionalization, and colloidal stability. ACS Nano 2012;6:6829-42.

32. Dianzani C, Zara GP, Maina G, Pettazzoni P, Pizzimenti S, Rossi F, et al. Drug delivery nanoparticles in skin cancers. BioMed Res Int 2014:2014:1-13. http://dx.doi.org/10.1155/2014/895986.

33. Lademann J, Patzelt A, Richter H, Antoniou C, Sterry W, Knorr F. Determination of the cuticula thickness of human and porcine hairs and their potential influence on the penetration of nanoparticles into the hair follicles. J Biomed Opt 2009;14:210-4.

34. Yu T, Malugin A, Ghandehari H. Impact of silica nanoparticle design on cellular toxicity and hemolytic activity. ACS Nanol 2011;5:5717-28.
35. Sharmiladevi S, Priya AS, Sujitha MV. Synthesis of mesoporous silica nanoparticles and drug loading for gram positive and gram negative bacteria. Int J Pharm Pharm Sci 2016;8:196-201.

36. Braun K, Pochert A, Linden M, Davoudi M, Schmidtchen A, Nordstrom $\mathrm{R}$, et al. Membrane interactions of mesoporous silica nanoparticles as carriers of antimicrobial peptides. J Colloid Interface Sci 2016;475:161-70.

37. Morry J, Ngamcherdtrakul W, Gu S, Goodyear SM, Castro DJ, Reda MM, et al. Dermal delivery of HSP47 siRNA with NOX4modulating mesoporous silica-based nanoparticles for treating fibrosis. Biomaterials 2015;66:41-52.

38. Ahmadi E, Dehghannejad N, Hashemikia S, Ghasemnejad M, Tabebordbar H. Synthesis and surface modification of mesoporous silica nanoparticles and its application as carriers for sustained drug delivery. Drug Delivery 2014;21:164-72.

39. Musso GE, Bottinelli E, Celi L, Magnacca G, Berlier G. Influence of surface functionalization on the hydrophilic character of mesoporous silica nanoparticles. Physical Chem Chem Phys 2015;17:13882-94.

40. Ngo MA, O'Malley M, Maibach HI. Perspectives on percutaneous penetration of nanomaterials. In Nanotechnology in Dermatology. New York, NY: Springer New York; 2013. p. 63-86.

41. Boonen J, Baert B, Lambert J, De Spiegeleer B. Skin penetration of silica microparticles. Die Pharmazie 2011;66:463-4.

42. Nohynek GJ, Lademann J, Ribaud C, Roberts MS. Grey goo on the skin? nanotechnology, cosmetic and sunscreen safety. Crit Rev Toxicol 2007;37:251-77.

43. Eskandar NG, Simovic S, Prestidge CA. Chemical stability and phase distribution of all-trans-retinol in nanoparticle-coated emulsions. Int J Pharm 2009;376:186-94.

44. Khan MA, Wallace WT, Islam SZ, Nagpure S, Strzalka J, Littleton $\mathrm{JM}$, et al. Adsorption and recovery of polyphenolic flavonoids using $\mathrm{TiO}_{2}$-functionalized mesoporous silica nanoparticles. ACS Appl MaterInterfaces 2017;9:32114-25.

45. Scalia S, Franceschinis E, Bertelli D, Iannuccelli V. Comparative evaluation of the effect of permeation enhancers, lipid nanoparticles and colloidal silica on in vivo human skin penetration of quercetin. Skin Pharmacol Physiol 2013;26:57-67.

46. Ngo MA, O'Malley M, Maibach HI. Percutaneous absorption and exposure assessment of pesticides. J Appl Toxicol 2010;30:91-114.

47. Götz C, Pfeiffer R, Tigges J, Ruwiedel K, Hubenthal U, Merk HF, et al. Xenobiotic metabolism capacities of human skin in comparison with a 3D epidermis model and keratinocyte-based cell culture as in vitro alternatives for chemical testing: activating enzymes (Phase I). Exp Dermatol 2012;21:358-63.

48. Jadhav SA, Cabanas MV, Manzano M, Vallet-Regi M. Controlled post-synthesis grafting of thermoresponsive poly(Nisopropylacrylamide) on mesoporous silica nanoparticles. Pol Adv Technol 2015;26:1070-5.

49. Paris JL, Cabanas MV, Manzano M, Vallet-Regi M. Polymergrafted mesoporous silica nanoparticles as ultrasoundresponsive drug carriers. ACS Nanol 2015;9:11023-33.

50. Soto RJ, Yang L, Schoenfisch MH. Functionalized mesoporous silica via an aminosilane surfactant ion exchange reaction: controlled scaffold design and nitric oxide release. ACS Appl Mater Interfaces 2016;8:2220-31.

51. Feinle A, Leichtfried F, Straber S, Husing N. Carboxylic acidfunctionalized porous silica particles by a co-condensation approach. J Sol Gel Sci Technol 2017;81:138-46.

52. Croissant JG, Cattoen X, Man MWC, Durand J, Khasab NM. Syntheses and applications of periodic mesoporous organosilica nanoparticles. Nanoscale 2015;7:20318-34.

53. Croissant JG, Fatieiev Y, Khashab NM. Degradability and clearance of silicon, organosilica, silsesquioxane, silica mixed oxide, and mesoporous silica nanoparticles. Adv Mater 2017;29:1604634.

54. Zhang Y, Hu L, Yu D, Gao C. Influence of silica particle internalization on adhesion and migration of human dermal fibroblasts. Biomaterials 2010;31:8465-74.

55. Crosera M, Bovenzi M, Mania G, Adami G, Zanette C, Florio C, et al. Nanoparticle dermal absorption and toxicity: a review of the literature. Int Arch Occup Environ Health 2009;82:1043-55.

56. Hudson SP, Padera RF, Langer R, Kohane DS. The biocompatibility of mesoporous silicates. Biomaterials 2008;29:4045-55.

57. Pandey P, Dahiya M. A brief review on inorganic nanoparticles. J Cri Rev 2016;3:18-26. 